D0I: 10.12957/demetra.2015.16095

\title{
Abordagem terapêutica da obesidade: entre conceitos e preconceitos
}

\section{Therapeutic approach of obesity: between concepts and prejudices}

Lucas Vieira Francisco

Rosa Wanda Diez-Garcia'

1 Universidade de São Paulo, Departamento de Clínica Médica, Faculdade de Medicina de Ribeirão Preto. PráticA - Laboratório de Práticas e Comportamento Alimentar, Nutrição e Metabolismo. Ribeirão Preto-SP, Brasil.

Correspondência / Correspondence Lucas Vieira Francisco

Av. dos Bandeirantes, 3900 - Campus

Universitário Monte Alegre CEP: 14040-902 - RibeirãoPreto-SP

E-mail: I.vieira.francisco@gmail.com

\section{Resumo}

A obesidade, historicamente, é carregada de significados, produto de uma construção histórica de valores e crenças aliados a contextos sociais e culturais que moldaram o conceito que temos hoje. Com o processo de medicalização, a obesidade passou a ser encarada como uma doença que precisa ser combatida e, com isso, certos julgamentos e críticas ao paciente com obesidade são disseminados, estabelecendo julgamentos que repercutem nos mais diversos aspectos da vida do indivíduo com excesso de peso. Por estarem imersas nesse contexto cultural, as equipes de saúde apresentam um padrão de comportamento similar ao da população geral. Vista como uma escolha individual, a obesidade se associa a preguiça, falta de força de vontade pessoal, entre outros atributos que contribuem para sua rejeição - forças históricas e dinâmicas que compõem a representação da obesidade. Entender os mecanismos e expressões do estigma da obesidade deve ser parte da formação de profissionais de saúde, estando atentos para evitar a recorrência do estigma, o que, devido às forças da pressão social, deve ser um exercício imposto no cotidiano profissional. Explorar as relações entre conceitos e preconceitos associados à obesidade é objetivo deste ensaio para subsidiar uma reflexão sobre a abordagem terapêutica da obesidade.

Palavras-chave: Obesidade. Estigma Social. Preconceito. 


\section{Abstract}

Obesity is historically full of meanings, product of a historical values and beliefs combined with social and cultural contexts that shaped the concept we have today. With the process of medicalization, obesity is regarded as a disease that needs to be fought and, therefore, certain judgments and criticisms of the patient with obesity are widespread, establishing judgments that impact in various aspects of overweight individuals. Immersed in this cultural context, health teams have a similar behavior pattern to the general population. Seen as an individual choice, obesity is associated with laziness, lack of personal willpower, among other attributes that contribute to their rejection - historical forces and dynamics that make up the representation of obesity. Understanding the mechanisms and obesity stigma of expressions should be part of the training of health professionals, avoiding the recurrence of stigma, which due to social pressure must be imposed on an exercise professional routine. This essay aims to explore the relationships between concepts and prejudices associated with obesity, subsidizing a reflection on the therapeutic approach to obesity.

Key words: Obesity. Social Stigma. Prejudice.

\section{Introdução}

A abordagem terapêutica da obesidade tem se detido no modelo biológico do qual derivam estratégias que são insuficientes para dar conta da complexidade implícita na sua determinação e no convívio com a obesidade e seus enfrentamentos.

A maneira pela qual as pessoas explicam a doença, suas causas e consequências em diferentes contextos, culturas e grupos sociais, incluindo o fenômeno biológico e sua relação com o social, ajuda a entender a experiência de viver uma dada condição, no caso, a de "estar obeso". A Antropologia Médica, ramo de estudo no qual as ciências sociais e naturais dialogam na compreensão de um determinado fenômeno, fundamenta esta argumentação. ${ }^{1}$ Pode-se dizer que é um ramo de estudo no qual as ciências sociais e naturais dialogam na compreensão de determinado fenômeno.

Os valores, costumes e crenças relacionados com determinada doença são partes de uma cultura e um contexto mais amplo e não podem ser estudados isoladamente, ou seja, não é possível entender como um indivíduo percebe uma doença, dor ou até mesmo a morte, sem entender o tipo 
de cultura em que ele está imerso. Também se faz necessário entender como a saúde, a doença e o processo de adoecimento são organizados socialmente, como por exemplo, os modos pelos quais as pessoas são categorizadas como doentes, ou como apresentam sua doença para os outros e os atributos que serão designados para ela. ${ }^{1}$

Dentre as doenças mais comum da atualidade, a obesidade tem papel central nesse cenário. Mundialmente, mais de 312 milhões de pessoas são obesas e mais de um bilhão apresentam sobrepeso. ${ }^{2}$ Na revisão sistemática realizada por Marie e colaboradores ${ }^{3}$ em 2014, que avaliou 1.769 artigos ao redor do mundo, pode-se concluir que entre 1980 e 2013, a proporção de excesso de peso em adultos aumentou de 18,9\% para 36,9\% em homens e $29,8 \%$ para $38 \%$ em mulheres, passando de 857 milhões de indivíduos para 2,1 bilhões em 2013. Em alguns países estudados, a prevalência de obesidade ultrapassa 50\%. Entretanto, apesar das taxas de obesidade e sobrepeso aumentarem nos últimos anos em todo o mundo, a discriminação de pessoas com obesidade também aumenta. Segundo a National Survey of Midlife Development, nos Estados Unidos, em dez anos aumentou cerca de 66\%; passando de 7\% em 1995/96 para 12\% em 2004/2006 a discriminação vinculada ao excesso de peso. ${ }^{4}$

O processo da medicalização, definido por Conrad ${ }^{5}$ como a atribuição de problemas tradicionalmente vistos como não médicos transformados em problemas médicos e, consequentemente, tratados em termos de disfunção e doença, participa do modo como a cultura lida com o doente e com a doença e, mais especificamente, contribui para determinar o que é doença e doente. A medicalização da obesidade substitui causas morais tradicionalmente construídas, por razões médicas, ao rotulá-la como "doença". Cria-se uma justificativa para "lutar" contra ela e o dever de ajudar e tratar sem a culpa do olhar moralizador, criando um pretexto para que todos possam atacar o paciente obeso e encarar isso como uma tentativa de combate à obesidade. ${ }^{6}$

Por sua importância global e complexidade de causas e tratamento, a obesidade merece ser vista e discutida além de seu aspecto biológico. Explorar as relações entre conceitos e preconceitos associados a obesidade é objetivo deste ensaio, para subsidiar uma reflexão sobre a abordagem terapêutica da obesidade.

\section{Algumas consideraçóes históricas conceituais sobre a obesidade}

A obesidade é carregada de símbolos e valores construídos ao longo de anos de história, esses significadosque começam na origem da própria palavra, que etimologicamente provém do latim "obedere", formada pelas raízes "-ob" (sobre, o que engloba) e "-edere"(comer). Portanto "aquele que come em excesso", sendo vinculado como aquele que tem atitudes fora do aceitável e dito como "anormal" por aquele grupo. ${ }^{7}$ 
Mantzoros $^{8}$ discorre sobre alguns marcos da história da obesidade ao longo dos anos. A primeira descrição da obesidade data da Era Paleolítica (cerca de 25.000 anos atrás), com uma série de artefatos descobertos ao longo de toda Europa. Esses artefatos variam de tamanho e forma, mas apresentam em comum figuras com grandes seios, sendo a mais importante a "Vênus de Willendorf", mostrando não apenas a existência da obesidade, como também talvez um símbolo projetado e idealizado de fertilidade e saúde. Durante o período Neolítico (8.000 a 5.500 anos AC) vários outros artefatos foram descobertos com fartos seios e grandes quadris e barrigas, representando as "Deusas-Mães".

Já na cultura egípcia (2.500 AC), achados mostram que a obesidade nada mais era do que um commodity dos ricos, apesar de que algumas múmias obesas tinham lugar de destaque entre os afortunados. Na China Antiga, já se tentava tratar a obesidade através da inserção de agulhas no corpo, como atualmente temos a acupuntura, na tentativa de reduzir o apetite e causar redução do peso e do tamanho corporal. Os tibetanos, seguindo essa crença da tradição chinesa, acreditavam que a causa da obesidade era o excesso de peso e a única forma de tratá-la seria reduzir o consumo de alimentos, e já descreviam em seus textos o fato de que "excesso de alimentos [...] causa doenças e menor duração da vida”.

Durante as Eras Clássicas (500 AC a 100 DC), a medicina greco-romana se dedicou a entender e caracterizar a obesidade. Hipócrates fez uma série de medidas rígidas para o tratamento da obesidade, mostrando que dieta e "mudanças de estilo de vida" são orientações recorrentes na história da obesidade:

Pessoas obesas e aqueles que desejam perder peso deveriam realizar trabalho pesado antes do alimento. Refeições devem ser feitas após o exercício e ainda sentindo a dor da fadiga e sem nenhum outro refresco antes da refeição, além de vinho diluído e levemente gelado [...]. Eles devem, além disso, comer apenas uma vez ao dia, não tomar banhos, dormir numa cama dura e andar nus o máximo possível. ${ }^{9}$

Galen, grande médico grego da mesma época, deu um passo no movimento de classificação da obesidade ao caracterizá-la como "moderada" e "excessiva".

Na Era Científica, por volta de 1614, dando continuidade a essa tentativa de classificar a pessoa com obesidade e influenciado pelos princípios de Galileu sobre a mensuração, Santorino elaborou uma escala que permitiu aos médicos avaliar a extensão da obesidade, com medidas corporais e evoluções longitudinais de melhora ou piora do peso. ${ }^{8}$ Esse movimento reforçou a ideia da classificação e rotulação do obeso de acordo com seu peso em faixas numéricas.

Adolphe Quetelet, preocupado com definir as características do "homem normal” e encaixálo numa distribuição normal, publicou em 1835 um livro compilando seus estudos revendo as 
mudanças de altura e peso pela idade e também a relação entre altura e peso para predizer índices de obesidade, criando assim o índice Quetelet. Em 1972, esse índice passou a ser chamado como conhecemos hoje, de índice de massa corporal (IMC), por Ancel Keys. ${ }^{10}$

Na Conferência de 1985 sobre as implicações para a saúde da obesidade, o Instituto Nacional de Saúde concordou em referir a obesidade como doença. O International Statistical Classification of Diseases and Related Health Problems fornece internacionalmente os códigos relativos a cada doença, bem como sinais, sintomas e causas, tem duas inscrições para a obesidade (278.00 e 278.01, "obesidade inespecífica" e "obesidade mórbida", respectivamente) ${ }^{11}$. Em 1998, A Organização Mundial da Saúde declarou que a obesidade é a principal doença não transmissível do século XXI. ${ }^{12}$

Lawrence, 2004, ${ }^{13}$ ao analisar os documentos e notícias a partir de 1985 , refere que a partir de 1992 as aparições na mídia da obesidade aumentam exponencialmente, sobretudo relacionadas com palavras como junk food, fast food e meio ambiente, refletindo a maior atenção sobre o assunto. A obesidade deixa de ser vista apenas como um fator de risco para outras doenças e passa a ser entendida como uma doença crônica, pelo balanço energético positivo entre ingestão e gasto energético. Mais recentemente, foram agregados nesse conceito os aspectos hormonais e inflamatórios do tecido adiposo na obesidade, mudando assim seu estado epistêmico e cerne do problema que passa a focar na gordura corporal..$^{14}$

\section{Conceitos implícitos e explícitos na obesidade}

Frente aos estudos das consequências sanitárias derivadas do excesso de peso, nas últimas décadas a literatura se deteve também no impacto social da obesidade, evidenciando que seus efeitos ultrapassam a questão puramente fisiológica.

Nas revisões de Puhl em $2001{ }^{15}$ e $2009,{ }^{16}$ fica claro o impacto do preconceito nos vários aspectos da vida diária do paciente com obesidade. Salários, promoções no trabalho e contratações são menores entre os sujeitos com excesso de peso. Eles são vistos como menos disciplinados e menos eficientes quando comparados com não obesos. Estudo longitudinal de oito anos com jovens adultos mostrou que mulheres obesas chegam a ganhar US\$ 6.000 menos do que as não obesas. ${ }^{17}$

Quando atitudes negativas em relação ao excesso de peso existem entre profissionais de saúde, podem afetar o julgamento clínico e dificultar o acesso do paciente aos serviços de saúde. ${ }^{16}$ Exemplo disso é a menor probabilidade de mulheres com excessos de peso se submeterem a exames pélvicos como Papanicolau ou mamografias, quando comparadas com mulheres eutróficas, mostrando a obesidade como barreira ao acesso a serviços de cuidados preventivos. ${ }^{18}$ A quantidade de exames solicitados e tempo de consulta também foram menores entre os pacientes com obesidade. Por 
exemplo, a consulta destes foi nove minutos mais curta que com os demais pacientes. ${ }^{19}$ Estudos também sinalizaram diferenças na abordagem do paciente obeso entre especialidades médicas e categorias de profissionais de saúde..$^{20,21}$

Ao avaliar atitudes de 620 médicos americanos em relação ao paciente obeso, ${ }^{22}$ verificou-se que $50 \%$ ou mais dos pesquisados o viam como desajeitados, feios, não atraentes e inadequados. Cerca de $30 \%$ a $45 \%$ caracterizavam esse tipo de paciente como desleixado, preguiçoso e com pouca força de vontade; e $9 \%$ e $3 \%$ indicaram os indivíduos obesos como desagradáveis e desonestos, respectivamente.

Estudo realizado com 250 médicos entre clínicos gerais, pediatras e psiquiatras concluiu que mais de $40 \%$ tinham uma atitude negativa com o paciente obeso; apenas $56 \%$ se sentiam qualificados para tratar a obesidade e $46 \%$ deles referiram que o tratamento tinha sucesso. ${ }^{23} \mathrm{E}$ na revisão sobre crenças e práticas sobre a obesidade entre profissionais de saúde, ${ }^{24}$ concluiu-se que o tratamento da obesidade é uma dificuldade para a maioria dos médicos estudados. Entre $74 \%$ e 96,4\% afirmaram que tratar a obesidade não é fácil, principalmente ao estabelecer metas a longo prazo, o que os leva a sentimentos de limitação e frustração.

Os problemas apontados sinalizam dificuldades no enfrentamento da obesidade, que extrapolam os muros institucionais. Segundo Kirk, ${ }^{25}$ essas tensões culminam na manutenção de um sistema médico vigente que não consegue tratar de maneira efetiva o excesso de peso.

Gracia-Arnaiz, ${ }^{26}$ ao comparar a visão de profissionais de saúde e jovens pacientes com obesidade através de estudo etnográfico realizado na Catalunha, Espanha, concluiu que a perspectiva diverge e converge em alguns pontos. Os jovens pacientes são considerados vítimas de uma sociedade permissiva e de abundâncias e, ao mesmo tempo, culpados por não seguirem recomendações médicas. E num processo duplo de medicalização e moralização, o paciente deve abandonar seus "maus" hábitos alimentares para adquirir um novo conjunto de hábitos "normais", seguindo orientações nutricionais e cientificamente estabelecidas, aprendendo a comer de "modo civilizado".

Estudo com 389 profissionais no Annual Meeting of North American Association for the Study of Obesity, realizado em Quebec, Canadá mostrou que, mesmo dentre os grupos “especializados” e interessados na obesidade, existem níveis consideráveis de preconceito, próximos ao da população em geral. Foi encontrada uma visão do paciente obeso como mais preguiçoso, estúpido e de menor valor. As mulheres mais jovens e mais magras foram as mais rígidas nesse julgamento. ${ }^{27}$

Não há dúvida de que o estigma sofrido pelo paciente obeso tem impacto negativo na sua saúde emocional e afeta seu bem-estar, colaborando com a baixa autoestima, que pode inclusive contribuir para a manutenção e ganho de peso. ${ }^{28,29}$ 


\section{Reflexão sobre a abordagem terapêutica da obesidade}

Os indivíduos que exercem a medicina moderno-cientifica formam um grupo com seus valores, regras, hierarquias e teorias próprias sobre a doença. Durante o processo de formação acadêmica na área da saúde, gradativamente vai se adquirindo um recorte sobre a visão de doença que perdurará ao longo de sua carreira. ${ }^{1}$ Dentre esses valores e ideais está a base da racionalidade científica, ou seja, um pensamento baseado e medido objetivamente; "fatos clínicos" passam a ser os motivos de reais investigações na maioria das vezes. Desse modo, vai acontecendo um processo crescente de definições numéricas para classificar estados de saúde e doença - ou seja, parâmetros físicos e bioquímicos (peso, altura, contagem de substâncias sanguínea, níveis hormonais) - que produzem faixas numéricas para o que é considerado normal ou saudável. Portanto, a doença e o anormal passam a ser o desvio dessa faixa para cima ou para baixo. ${ }^{1}$

Mais do que um sistema de ideias, práticas e modelos científicos, o exercício profissional é constituído por um sistema simbolicamente construído, expressando valores, crenças e questões morais de determinada sociedade num contexto histórico. Nas últimas décadas, nas sociedades industrializadas, esse aspecto moral da medicina vem ganhando mais força com o declínio dos valores religiosos, visto que antigamente um infortúnio era causado por uma falha moral e hoje uma doença é causada por um estilo de vida não saudável. Os antigos pecados capitais de gula e preguiça foram substituídos pelos novos conceitos de "alimentação inadequada" e "sedentarismo".

Com esses novos valores e julgamentos morais atrelados à doença, as patologias que são atribuídas a escolhas pessoais vêm carregadas da responsabilidade que recai sobre o próprio paciente podendo levar ao estigma destes. Em uma perspectiva social, o estigma vinculado a certas doenças acarreta sofrimento adicional aos pacientes. Eles sentem responsabilidade e culpa frente à sociedade, incorporando juízos morais sobre as circunstâncias em que a enfermidade foi provocada. Sofrem discriminações em diversos âmbitos da vida, como os exemplos citados acima.

A obesidade, para Bartolomé, ${ }^{7}$ representa um signo visível de "transgressão normativa”, ou seja, a pessoa com obesidade é vista como um indivíduo que contradiz um modelo ideal da sociedade atual, que é a obsessão pelo culto ao corpo, estética e magreza. Esta se manifesta no medo de engordar e de não ter um corpo socialmente aceito. O corpo magro reúne num protótipo as características consideradas perfeitas. Preocupações e inconvenientes são consequências para aqueles que não estão nesses limites socialmente estabelecidos.

A "lipofobia" ou "gordurofobia" 30 é um fenômeno recente nas sociedades ocidentais, caracterizada como uma rejeição sistemática à gordura e ao medo de se tornar obeso. Esses padrões de beleza foram modificados dependentes de contextos sociais, históricos, culturais e geográficos, numa constante luta entre o aceito, desejado e belo versus o rejeitado, criticado e o feio. Nos primórdios da humanidade, a obesidade foi tida como sinônimo de fertilidade e saúde, assim 
como na Idade Média, para depois ser confrontado com ideologias judaico-cristãs da moderação e restrição. Com isso, desde a época renascentista, nas sociedades ocidentais industrializadas, a figura obesa foi perdendo seu valor.

A valorização do corpo magro é também atrelada à conscientização terceiro-mundista e à crítica ao capitalismo - do mesmo modo que o capitalista acumula o capital e recursos, o obeso acumula energia sob a forma de gordura corporal. No imaginário coletivo tradicional do "anticapitalismo" da década de 1960, representava-se o patrão como um indivíduo barrigudo, com um charuto e notas saindo da cartola. A figura do obeso, segundo os caricaturistas, passa a denunciar o sistema capitalista e as desigualdades alimentares entre colônias e pós-colônias do Hemisfério Sul versus o Norte. A gordura passa a ser moralmente incorreta sob essa perspectiva, significando egoísmo daquele que não aceita a lógica de redistribuição, enquanto a magreza se torna um sinal de integridade moral. ${ }^{6}$

Gracia-Arnaiz ${ }^{31}$ refere que os hábitos alimentares atuais contribuíram para promover o discurso de "civilizar o apetite". Com a evolução dos conhecimentos científicos que mostram a conexão entre dieta, doença e saúde, retomam-se certos ideais de autocontrole, restrição e moderação antigamente usados para a alimentação, transformando assim a questão da alimentação em "fábricas de moral", moldando ideais de corpo e práticas alimentares e comportamentais.O corpo ideal e desejado se torna mais do que apenas uma imagem e um símbolo estético, e passa a ser uma questão de poder. ${ }^{32}$ No contexto de uma sociedade lipofóbica, o tamanho corporal serve para classificar pessoas tanto no aspecto biológico e de saúde quanto na questão moral e de personalidade. ${ }^{31}$

Enquanto o corpo magro é visto como moral, médico e esteticamente desejável e sinônimo de moderação, autocontrole e empenho, o corpo obeso significa preguiça, gula e doença. ${ }^{16}$ Pode representar uma falha pessoal, social e médica daquele indivíduo, tornando a perda de peso mais uma tentativa de escapar das pressóes sociais e emocionais de ser obeso do que uma preocupação real com a saúde. ${ }^{31}$

O "estar de dieta" é resultado das inúmeras pressões existentes, tornando-se uma condição permanente e desejável, envolvendo processos de moralização e medicalização e, paradoxalmente, trocando um modelo de obsessão da alimentação por outro modelo biomédico nutricional que dita o que deve ou não deve ser comido. ${ }^{33}$

Apesar dos inúmeros estudos mostrando as diversas causas da obesidade, como fatores genéticos, hormonais e metabólicos, ainda sobressai a hipervalorização das causas pessoais do excesso do acumulo de gordura corporal. ${ }^{34}$ Pessoas com obesidade ainda são rotuladas como os grandes comedores, que não se controlam e comem mais do que deveriam, refletindo uma interpretação moralista e carregada de preconceito. E como vimos, os profissionais de saúde, por estarem imersos nesse jogo de forças, acabam agindo da mesma forma que a população em geral. ${ }^{26}$ 
Na relação entre as crenças simbólicas com a rejeição dos obesos, Crandall ${ }^{35}$ propõe serem as atitudes antigordura resultantes de uma visão de mundo influenciada por ideologias derivadas de uma ética de trabalho puritana, do livre arbítrio, da crença num mundo justo e da noção de que as pessoas têm o que merecem. Ele discorre que essas forças, juntas e em diferentes proporções, se tornam justificativas para o estigmatizador atribuir causas controláveis aos outros, tendendo a culpar o obeso por seu próprio peso. Ele também conclui que o racismo e as atitudes antigordura têm uma raiz ideológica parecida, ou seja, são baseadas nas ideologias e valores conservadores, autoritários e que rejeitam o diferente. As atitudes antigordura não possuem uma supressão social tão forte e bem estruturada quanto o racismo, mas são comuns e explícitas no cotidiano, provavelmente por serem um padrão novo em nossa sociedade.

Apesar de o preconceito da obesidade vir sendo demonstrado por décadas pelos cientistas sociais, ainda parece estar em um estágio de maturidade muito aquém das necessidades. Esse preconceito se compara ao estágio do racismo de 50 anos atrás: manifesto, expresso e amplamente difundido no cotidiano. ${ }^{35}$

O corpo obeso como reflexo de características pessoais é tão forte que, mesmo após a perda de peso, o estigma pode permanecer. Vartanian, ${ }^{36}$ comparando histórico de cirurgia com dieta e exercício para perda de peso, mostrou que estudantes, ao saberem do histórico de um paciente, julgam-no mais preguiçoso, desleixado e menos competente, sociável, atrativo e saudável. Ao saberem que um indivíduo foi obeso e emagreceu através de cirurgia, consideram que ele escolheu "o caminho fácil”, que não requer esforço.

Outra perspectiva é a obesidade ser vista como produto da incapacidade de alcançar um equilíbrio do gasto energético. Nessa visão individualista, o paciente é visto como alguém que não consegue tomar as decisões certas sobre o que ingere e o que gasta de energia, incapaz de gerir o próprio corpo e tomar decisões racionais. ${ }^{15,16,24}$ Desse modo, as atribuições sociais atreladas às causas da obesidade reforçam a manifestação do estigma, por se pensar que o indivíduo obeso é o responsável pela sua própria doença, devido à falta de vontade e autodisciplina, o que implica responsabilidade pessoal como peça central no processo de estigmatização do paciente obeso.

Com o aumento da obesidade, os gastos públicos para tratá-la também aumentam na mesma escala. Alguns grupos reclamam de gastos públicos em saúde com patologias causadas por falta de autocuidado, isto é, pacientes obesos são rotulados como "aqueles que não se cuidam" ou que "não querem se cuidar e gastam dinheiro público para isso", sendo, com isso, cada vez mais punidos por esse dilema moral de culpa individual.

A mídia é peça central para a transmissão de valores, ideais e, consequentemente, preconceitos. Ao revisar a relação da mídia com a obesidade, $\mathrm{Puhl}{ }^{16}$ constatou que personagens obesas nas mídias de entretenimento normalmente são atreladas a um comportamento alimentar estereotipado ou 
com características cômicas e ridículas, associadas a falas seguidas por risadas da plateia, muitas vezes descritas como pouco atraentes, burras e infelizes. Nas mídias de notícias, isso também pode ser constatado pelo modo como é dado destaque na responsabilidade pessoal da obesidade, enfocando causas e soluções no nível individual, como comer em excesso e como mudar sua própria dieta. Isso reflete o entendimento público da obesidade, mascarando outras dimensões dessa doença e reforçando a culpa individual e os preconceitos. Por outro lado, há sites e fóruns com conteúdo de discussão, incentivo e orientação que podem ajudar a inverter essas abordagem pejorativa, conforme estudo de Wen-ying. ${ }^{37}$

\section{Considerações finais}

O modo como percebemos a obesidade é filtrado por seu percurso histórico e por mecanismos de "normalização" do corpo e do comportamento. Essas inúmeras forças constroem a imagem da obesidade e do paciente obeso, de forma dinâmica e fluida.

A obesidade é reconhecida como epidemia mundial multifatorial, mas isso não tem sido suficiente para influenciar seu tratamento. Muitas vezes, o reconhecimento de fatores que vão além do âmbito da saúde, como a influência ambiental, mantém os aspectos aqui abordados em um campo nebuloso e complexo.

Entender os mecanismos e expressões do estigma da obesidade deve ser parte da formação de profissionais de saúde. Manter-se atentos para evitar a recorrência desse estigma, dadas as forças da pressão social, deve ser um exercício imposto no cotidiano profissional.

\section{Agradecimentos}

Agradecemos ao apoio do CNPq - Conselho Nacional de Desenvolvimento Científico e Tecnológico.

\section{Referências}

1. Helman CG. Cultura, saúde \& doença. 4 ed. Porto Alegre: Artmed; 2003.

2. Lopez AD, Mathers CD, Ezzati M, Jamison DT, Murray CJ. Global and regional burden of disease and risk factors, 2001: systematic analysis of population health data. The Lancet 2006; 367(9524):1747-1757.

3. Ng, Fleming T, Robinson M, Thomson B, Graetz N, Margono C, et al. Global, regional, and national prevalence of overweight and obesity in children and adults during 1980-2013: a systematic analysis for the Global Burden of Disease Study 2013. The Lancet 2014; 384(9945):766-81. 
4. Mujahid MS, Diez Roux AV, Borrell LN, Javier Nieto F. Cross Sectional and Longitudinal Associations of BMI with socioeconomic characteristics. Obesity Research 2005; 13(8):1412-1421.

5. Conrad P. Medicalization and social control. Annual Review of Sociology 1992; 18:209-232.

6. Poulain JP. Sociologia da obesidade. São Paulo: Editora Senac; 2013.

7. Bartolomé MD, Guzmán JL. La estigmatización social de la obesidad. Cuadernos de Bioética 2014; 25:273-284.

8. Mantzoros CS. Obesity and diabetes. New Jersey: Human Press; 2006.

9. Precope J. Hippocrates on diet and hygiene. London: Zeno; 1952 apud Mantzoros CS. Obesity and diabetes. New Jersey: Human Press; 2006.

10. Eknoyan G. Adolphe Quetelet (1796-1874): the average man and indices of obesity. Nephrol. Dial. Transplant 2008; 23:47-51.

11. World Health Organization. International classification of disease. 9 ed. Geneva: WHO; 1977.

12. World Health Organization. Obesity: preventing and managing the global epidemic. Working Group on Obesity. Geneva: WHO; 1998.

13. Lawrence RG. Framing obesity: the evolution of news discourse on a public health issue. Press Politics 2004; 9(3):56-75.

14. Kopelman PG. Obesity as a medical problem. Nature 2000; 404(6):635-643.

15. Puhl R, Brownell KD. Bias, Discrimination, and obesity. Obesity Research 2001; 9(12):788-805.

16. Puhl RM, Heuer CA. The stigma of obesity: a review and update. Obesity 2009; 17(5):941-964.

17. Pagan JA, Dávila A. Obesity, occupational attainment, and earnings. Social Science Quarterly 1997; 78(3):756-770.

18. Wee CC, McCarthy EP, Davis RB, Phillips RS. Screening for cervical and breast cancer: is obesity an unrecognized barrier to preventive care? Annals of Internal Medicine 2000; 132(9):697-703.

19. Helb MR, Xu J. Weighing the care: physicians' reactions to the size of a patient. International Jornal of Obesity 2001; 25:1246-1252.

20. Kristeller JL, Hoerr RA. Physician attitudes toward managing obesityl differences among six specialty groups. Preventive Medicine 1997; 26:542-549.

21. Budd GM et al. Health care professionals' atitude about obesity: an integrative review. Applied Nursing Research 2011; 24:127-137.

22. Foster GD, Wadden TA, Makris AP, Davidson D, Sanderson RS, Allison DB, et al. Primary care physicians' attitudes about obesity and its treatment. Obesity Research 2003; 11(10):1168-1177.

23. Jay M, Kalet A, Ark T, McMacken M, Messito MJ, Richter R, et al. Physicians' attitudes about obesity and their associations with competency and speciality: a cross-sectional study. BMC Health Services Research 2009; 9:106.

24. Teixeira FV, Pais-Ribeiro JL, Maia ARPC. Crenças e práticas dos profissionais de saúde face a obesidade: uma revisão sistemática. Rev. Assoc. Méd. Bras. 2012; 58(2):254-262. 
25. Kirk SFL, Preço SL, Penney TL, Rehman L, Lyons RF, Piccinini-Vallis H, et al. Blame, shame, and lack of support: a multilevel study on obesity management. Qualitative Health Research 2014; 24(6):780-800.

26. Gracia-Arnaiz M. Thou shalt not get fat: medical representations and self-images of obesity in a Mediterranean society. Health 2013; 5(7): 1180-1189.

27. Schwartz MB, Chambliss HO, Brownell KD, Blair SN, Billington C. Weight bias among health professionals specializing in obesity. Obesity Research 2003; 11(9):1033-1039.

28. Lewis S, Thomas SL, Sangue RW, Castelo DJ, Hyde J, Komesaroff PA. How do obese individuals perceive and respond to the different types of obesity stigma that they encounter in their daily lives? a qualitative study. Social Science \& Medicine 2011; 73(9):1349-1356.

29. Brewis AA. Stigma and the perpetuation of obesity. Social Science \& Medicine 2014; 118: 152-158.

30. Saguy A, Ward A. Coming out as fat: rethinking stigma. Social Psychology Quarterly 2011; 20:1-23.

31. Gracia-Arnaiz M. Comer bien, comer mal: la medicalización del comportamiento alimentar. Salud Pública de México 2007; 49(3):236-242.

32. Saint Pol T. The desirable body. Paris: Presses Universitaires de France; 2010.

33. Gracia-Arnaiz M. Fat bodies and thin bodies. Cultural, biomedical and market discourses on obesity. Appetite 2010; 55:219-225.

34. Friedman JM. Modern science versus the stigma of obesity. Natural Medicine 2004; 10(6):563-569.

35. Crandall CS. Prejudice against fat people: ideology and self-interest. Journal of Personality and Social Psychology 1994; 66(5):882-894.

36. Vartanian LR, Fardouly J. The stigma of obesity surgery: negative evaluations based on weight loss history. Obesity Surgery 2013; 23:1545-1550.

37. Wen-ying SC, Prestin A, Kunath S. Obesity in social media: a mixed methods analysis. TBM 2014; 4(3):314-323.

Recebido: $15 / 4 / 2015$

Aprovado: 02/7/2015 